\title{
Neutrinos from Stellar Collapse: Comparison of the effects of three and four flavour mixings
}

\author{
Gautam Dutta, D. Indumathi, M. V. N. Murthy and G. Rajasekaran \\ The Institute of Mathematical Sciences, Chennai 600 113, India.
}

(December 17, 2018)

\begin{abstract}
We study the effect of non-vanishing masses and mixings among neutrino flavours on the detection of neutrinos from stellar collapse by a water Cerenkov detector. We consider a frame-work in which there are four neutrino flavours, including a sterile neutrino, whose mass squared differences and mixings are constrained by the present understanding of solar, atmospheric and laboratory neutrino detection. We also include the effects of high density matter within the supernova core. Unlike in the three flavour scenario, we find that the number of events due to the dominant process involving electron-antineutrinos changes dramatically for some allowed mixing parameters. Furthermore, contributions from charged-current scattering off oxygen nuclei in the detector can be considerably enhanced due to flavour mixing. We also present a comparison between the two possible scenarios, namely, when only three active neutrino flavours are present and when they are accompanied by a fourth sterile neutrino.
\end{abstract}




\section{INTRODUCTION}

In a recent paper [1] (hereafter referred to as I), we discussed in detail the signatures of 3 flavours of neutrinos from stellar collapse. The analysis was confined to Type II supernovae (which occur when the initial mass of the star is between 8-20 solar masses). Based on the work of Kuo and Pantaleone [2], where they include mixing among all three neutrino flavours, we found that the mixing between neutrino flavours leaves non-trivial signatures in the detector. The main conclusion of the paper was that the effect of mixing is to produce a dramatic increase in the events involving oxygen targets [3]. These will show up as a marked increase in the number of events in the backward direction with respect to the forward peaked events involving electrons as targets (more than $90 \%$ of which lie in a $10^{\circ}$ forward cone with respect to the supernova direction for neutrinos with energies $E_{\nu}>8 \mathrm{MeV}$ ). In the absence of any mixing, there will also be a few events in the backward direction due to CC scattering on oxygen targets. Furthermore, the observed $\bar{\nu}_{e} p$ events are the largest in number as well as least sensitive to the mixing parameters within the three flavour scenario. Hence they provide a direct test of the supernova models. Since the angular distribution of these events is approximately isotropic [10], they may be used to set the overall normalisation.

The above analysis was done assuming the standard mass heirarchy necessitated by the solar and atmospheric neutrino observations [5]. In the meantime, several authors have looked at the possible signatures of neutrinos and antineutrinos from supernova collapse for Super Kamiokande and Sudbury Neutrino Observatory(SNO). Dighe and Smirnov [6] have looked at the problem of reconstruction of neutrino mass spectrum in a three flavour scenario. These authors as also Chiu and Kuo [7] have compared the signatures in the standard mass heirarchy and inverted-mass heirarchy. While these papers and I incorporate the constraints from solar and atmospheric neutrino observations, the important question

of the mass limits that may be obtained from the observation of time delay has also been analysed by Choubey and Kar [8] (see also the review by Vogel [9]). For a recent review which also discusses aspects of locating a supernova by its neutrinos in advance of optical observation, see ref. [10].

In this paper, we continue the main theme of paper I, and extend the analysis to include a fourth sterile neutrino as well as discuss some aspects of three flavour mixing not discussed in detail in I. The main motivation to extend the analysis comes from the fact that such a sterile neutrino is probably required in order to explain the recent results from the LSND collaboration [11]. It is well known that an explanation of the solar and atmospheric neutrino puzzles involve two very different scales of mass-squared differences. As such, both of them cannot be explained unless one invokes at least three neutrino flavours. However, the scale required in order to understand the results from the LSND collaboration is different from both atmospheric and solar neutrino puzzles. It has therefore become necessary to introduce an extra-neutrino species, $\nu_{s}$, which however must remain sterile due to the LEP constraint on the Z-width.

The main feature of the analysis as emphasised in I-that the charged current (CC) events on oxygen nuclei (which show a preference to be in the backward direction) - is preserved both in three and four flavour scenarios. We also find that the signatures for three and four flavours are very distinct for some parameter ranges in the dominant isotropic events caused by electron antineutrino interactions with protons in water. 
The analysis presented in this paper follows closely the earlier analysis presented in I and therefore we will only reproduce the main outline and refer to I for some details. In Sect. II we give an outline of the framework and the mixing matrix as also matter effects on mixing. We then use this to obtain expressions for the fluxes reaching the detector in Sect. III. Sect. IV contains an analytic discussion of the signatures in the case of extreme adiabatic and extreme non-adiabatic mixing. In Sect. V we write down expressions for the event rates due to interactions between different neutrino flavours and scattering targets in the detector. Numerical results are presented in Sect. VI both for the spectrum and the integrated number of events. We present a summary and list our conclusions in Sect. VII. Appendix A gives details of the choice of mixing matrix that we have used in the paper.

\section{MIXING IN THE PRESENCE OF HIGHLY DENSE MATTER}

We briefly discuss mixing among four flavours of neutrinos (or antineutrinos) and compute the neutrino survival and conversion probabilities.

Unlike in the three flavour scenario, the addition of a sterile component forces us to prescribe a heirarchy of states in the mass eigenstate basis. If neutrino oscillation is the mechanism for the result from LSND, it is clear that the conversion of $\nu_{\mu} \rightarrow \nu_{e}$ is governed by a mass scale in the range of $0.1 \mathrm{eV}^{2}$ to $1 \mathrm{eV}^{2}$. As shown in Fig.1, we choose two doublets separated by this mass scale. In the lower doublet the mass-squared difference is given by the appropriate scale for the solution of the solar neutrino puzzle $\left(\delta m_{S}^{2}<10^{-5} \mathrm{eV}^{2}\right)$. Analogously, in the upper doublet it is the atmospheric neutrino mass scale $\left(\delta m_{A T M}^{2} \approx\right.$ $10^{-3} \mathrm{eV}^{2}$ ) that plays the crucial role. In principle the sterile neutrino may be in either of these doublets. However, if one believes that the atmospheric neutrino solution is driven by the conversion of $\nu_{\mu} \rightarrow \nu_{\tau}$, then the sterile neutrino should be in the lower doublet accounting for the solar neutrino deficit. While we take the sterile neutrino in the lower doublet, the final results are not crucially dependent on where we position the sterile neutrino as long as the two doublet scheme is adhered to.

The mixing matrix, which relates the flavour and mass eigenstates in the four flavour scenario has six angles and the CP-violating phases which we do not consider here. As is the convention, we denote the mixing angle in the lower doublet by $\omega$ and the mixing angle in the upper doublet by $\psi$. The resulting complicated mixing matrix involving six mixing angles is greatly simplified by application of the CHOOZ [12] constraint. The CHOOZ result implies that $\sin \theta \leq \epsilon$, where $\theta$ is either the (13) or (14) mixing angle and $\epsilon \leq 0.16$. We will therefore replace these angles by their maximum possible values allowed by CHOOz. Furthermore, we choose $\sin \theta_{13} \sim \sin \theta_{14} \sim \sin \theta_{23} \sim \sin \theta_{24} \sim \epsilon$. (The oscillation probabilities we are mainly interested in depend on the $\nu_{e}$ survival and conversion probabilities. These involve only $\theta_{13}$ and $\theta_{14}$ mixing angles. As such $\theta_{23}$ and $\theta_{24}$ may be kept arbitrary and have been fixed to $\epsilon$ for convenience. Note that the $\mathrm{CHOOZ}$ constraint corresponds to the replacement $\sin \phi \rightarrow \epsilon$ in the three flavour case analysed in I, where $\phi$ is the (13) mixing angle to which the $\mathrm{CHOOZ}$ constraint applies. This choice of hierarchy and mixings is consistent with already known data from various laboratory and atmospheric neutrino experiments. For more details on the constraints on mass squared differences and mixing angles from various experiments, see

Appendix A. Then the flavour eigenstates are related to the four mass eigenstates in vacuum (for both neutrinos and antineutrinos) through a unitary transformation, 


$$
\left[\begin{array}{l}
\nu_{e} \\
\nu_{s} \\
\nu_{\mu} \\
\nu_{\tau}
\end{array}\right]=U^{v}\left[\begin{array}{l}
\nu_{1} \\
\nu_{2} \\
\nu_{3} \\
\nu_{4}
\end{array}\right]
$$

where the superscript $v$ on the r.h.s. stands for vacuum. Within the two doublet scheme the $4 \times 4$ unitary matrix, $U^{v}$, may be written as

$$
U^{v}=\left(\begin{array}{cccc}
\left(1-\epsilon^{2}\right) c_{\omega} & \left(1-\epsilon^{2}\right) s_{\omega} & \epsilon & \epsilon \\
-\left(1-\epsilon^{2}\right) s_{\omega}-2 \epsilon^{2} c_{\omega} & \left(1-\epsilon^{2}\right) c_{\omega}-2 \epsilon^{2} s_{\omega} & \epsilon & \epsilon \\
\epsilon\left(s_{\omega}-c_{\omega}\right)\left(c_{\psi}+s_{\psi}\right) & -\epsilon\left(s_{\omega}+c_{\omega}\right)\left(c_{\psi}+s_{\psi}\right) & \left(1-\epsilon^{2}\right) c_{\psi}-2 \epsilon^{2} s_{\psi} & \left(1-\epsilon^{2}\right) s_{\psi} \\
\epsilon\left(s_{\omega}-c_{\omega}\right)\left(c_{\psi}-s_{\psi}\right) & -\epsilon\left(s_{\omega}+c_{\omega}\right)\left(c_{\psi}-s_{\psi}\right) & -\left(1-\epsilon^{2}\right) s_{\psi}-2 \epsilon^{2} c_{\psi} & \left(1-\epsilon^{2}\right) c_{\psi}
\end{array}\right)
$$

where $s_{\omega}=\sin \omega$ and $c_{\omega}=\cos \omega$, etc. The angles $\omega$ and $\psi$ can take values between 0 and $\pi / 2$. The mixing matrix given above is unitary up to order $\epsilon^{2}$. Since $\epsilon$ is a small parameter, we do not need to go beyond this order. Later on we will show that the survival and oscillation probabilities that we need are such that they do not depend on the mixing in the upper doublet, namely the angle $\psi$. For all practical purposes it can be set to any value, in particular, zero.

The masses of the eigenstates in vacuum are taken to be $\mu_{1}, \mu_{2}, \mu_{3}$ and $\mu_{4}$. In the mass eigenbasis, the (mass) ${ }^{2}$ matrix is diagonal:

$$
\begin{aligned}
M_{0}^{2} & =\mu_{1}^{2} I I+\left(\begin{array}{cccc}
0 & 0 & 0 & 0 \\
0 & \delta_{21} & 0 & 0 \\
0 & 0 & \delta_{31} & 0 \\
0 & 0 & 0 & \delta_{41}
\end{array}\right) \\
& =\mu_{1}^{2} I I+\Delta M_{0}^{2}
\end{aligned}
$$

where the mass squared differences are given by $\delta_{21}=\mu_{2}^{2}-\mu_{1}^{2}, \delta_{31}=\mu_{3}^{2}-\mu_{1}^{2}$ and $\delta_{41}=\mu_{4}^{2}-\mu_{1}^{2}$.

Without loss of generality, we can take $\delta_{21}, \delta_{31}$ and $\delta_{41}$ to be greater than zero; this defines the standard hierarchy of masses consistent with the range of the mixing angles, as specified above. Neutrino oscillation amplitudes are independent of the first term so we drop it from further calculation. In the flavour basis, therefore, the relevant part of the mass squared matrix has the form,

$$
\Delta M_{v}^{2}=U^{v} \Delta M_{0}^{2} U^{v \dagger}
$$

where $U^{v}$ is the mixing matrix in vacuum.

\section{A. Matter effects for neutrinos}

The relevant matter effects may be included by a modified mass squared matrix,

$$
\Delta M_{m}^{2}=\Delta M_{v}^{2}+\Delta M_{A},
$$

where the matter effects are included in, 


$$
\Delta M_{A}=\left(\begin{array}{cccc}
A_{1}(r) & 0 & 0 & 0 \\
0 & A_{2}(r) & 0 & 0 \\
0 & 0 & 0 & 0 \\
0 & 0 & 0 & 0
\end{array}\right)
$$

with the $A_{i}(r)$ given by

$$
A_{1}(r)=\sqrt{2} G_{F} N_{e}(r) \times 2 E
$$

and

$$
A_{2}(r) \approx \sqrt{2} G_{F} N_{n}(r) \times E
$$

which are proportional to the electron number density, $N_{e}(r)$, and neutron number density $N_{n}(r)$ respectively. We set $N_{e}(r) \simeq f_{e} N_{n}(r)$, where $f_{e}$ is the electron fraction which is less than one, in the supernova core. Here $r$ is the radial distance from the centre of the star. Note that we have subtracted the neutral current contribution to the active flavours in the interaction term. Since the sterile neutrino does not interact at all, we have added and subtracted the neutral current contribution; hence the appearance of $A_{2}$ in the masssquared matrix in matter. The detailed modifications due to matter effects are discussed in Appendix A of I.

As noted in I, the maximum value of $A_{1}$ occurs at the core and is approximately $2 \times$ $10^{7} \mathrm{E} \mathrm{eV}^{2}$, where $E$ is the neutrino energy in $\mathrm{MeV} . A_{2}$ is somewhat smaller. The modification due to the matter dependence is similar to the case of solar neutrinos, although, unlike in the case of solar neutrinos, all active flavours are produced in the supernova core.

It is clear that the mass squared matrix is no longer diagonalised by $U^{v}$ in the presence of matter; we therefore diagonalise $\Delta M_{m}^{2}$ in order to determine the matter-corrected eigenstates. The value of $A_{i}$ for energetic neutrinos (of a few $\mathrm{MeV}$ to tens of $\mathrm{MeV}$ ) in the core is several orders of magnitude greater than these mass-squared differences. The eigenvalue problem may thus be solved perturbatively, with the following hierarchy: $A_{i}$ (core) $\gg \delta_{41}>\delta_{31} \gg \delta_{21}$. As a result, the electron neutrino undergoes three Mikheyev, Smirnov and Wolfenstein (MSW) resonances [13]. In Fig. 2 we show schematically the level crossing pattern for neutrinos in the presence of matter. Note that in this case there are several level crossings because of the presence of the sterile neutrino.

\section{The adiabatic case}

Following the method outlined in I, we compute the eigenstates at production (almost all the active neutrino flux is produced in the core). Unless $\epsilon$ is extremely small, the propagation is adiabatic as may be shown by computing the Landau-Zener(LZ) probabilities at each resonance. While we include LZ probabilities in the actual numerical computation, we will first state the relevant probabilities in the case of adiabatic propagation of neutrinos produced in the supernova core. The average transition probability between two flavours $\alpha$ and $\beta$ is denoted by $P_{\alpha \beta}$ where $\alpha, \beta=e, \mu, \tau, s$. These are obtained by observing that almost all the $\nu$ flux is produced in the highly dense core. Here $A_{i} \approx 10^{7} \mathrm{eV}^{2}$, so one may take the 
extreme limit in which the density is infinite. Then the electron neutrino is produced as the highest mass eigenstate, that is,

$$
\left|\nu_{e}\right\rangle^{m}=\left|\nu_{4}\right\rangle^{m}
$$

A similar conclusion holds for the sterile partner in the lower doublet. In the case of adiabatic propagation the mass eigenstates produced in matter remain the same in vacuum. Therefore to a good approximation, the probabilities (of survival or transition into each other) for these

two flavours, $\nu_{e}$ and $\nu_{\mu}$, are just the overlap of the highest two mass eigen states with the corresponding vacuum eigen states, and are small, as can be seen from the relevant entries in the matrix, $U^{v}$, in eq. (2):

$$
P_{e e}=P_{e s}=P_{s e}=P_{s s}=\epsilon^{2},
$$

independent of other mixing angles and the mass squared differences. Note that $\epsilon^{2}$ is constrained by the limit set by the CHOOz collaboration and is small. Indeed in the purely adiabatic transition therefore the survival probability of the electron type neutrino is rather small. We also note that it is sufficient to know only these probabilities as long as the $\nu_{\mu}$ and $\nu_{\tau}$ are not separately detected. Furthermore, note that there is no initial flux for the sterile neutrino, but it can arise after oscillation due to mixing from other flavours.

\section{The non-adiabatic case}

A general non-adiabatic case is harder to discuss analytically. Since there are many crossings, one has now to consider non-adiabatic transitions at all these crossings. Because of the parametrization it is easy to see that non-adiabatic effects are introduced as a result of the values chosen for $\epsilon$ and $\omega$. The value of $\epsilon$ determines whether non-adiabatic jumps are induced at the upper resonances while the value of $\omega$ determines whether the non-adiabatic jump occurs at the lower resonance. This statement holds both for three and four flavours since in both cases the non-adiabaticity in the upper resonance(s) is controlled by $\epsilon$, apart from mass squared differences.

Note that for a large range of $\epsilon$, allowed by the CHOOZ constraint, the evolution of the electron neutrino is adiabatic. As a result the lower resonance does not come into the picture at all except when $\epsilon$ is very close to zero, where the jump probability abruptly changes to one. [2] The subsequent discussion is therefore relevant only when $\epsilon$ is almost vanishing which is not ruled out by the known constraints except in LSND. The extreme non-adiabatic case occurs when the nature of transitions at the lowest resonance which involves the crossing of the first and the second mass eigenstates is nonadiabatic. Partial non-adiabaticity results when the transition in the lowest resonance is adiabatic while in the upper resonances it is non-adiabatic. The probability of jump at the lower resonance is given by $P_{L}$ which is in general a function of $\omega$ and the mass squared difference $\delta m_{12}^{2}$. In our calculations we have used the form discussed in the appendix of I (see also [14]).

The relevant survival and oscillation probabilities for electron-neutrinos in the nonadiabatic case are given by,

$$
\begin{aligned}
& P_{e e}=\left(1-2 \epsilon^{2}\right)\left[\left(1-P_{L}\right) \sin ^{2} \omega+P_{L} \cos ^{2} \omega\right], \\
& P_{e s}=\left(1-2 \epsilon^{2}\right)\left[\left(1-P_{L}\right) \cos ^{2} \omega+P_{L} \sin ^{2} \omega\right],
\end{aligned}
$$




$$
\begin{aligned}
& P_{s s}=P_{e e}+2 \epsilon^{2}\left(1-2 P_{L}\right) \sin 2 \omega, \\
& P_{s e}=P_{e s}-2 \epsilon^{2}\left(1-2 P_{L}\right) \sin 2 \omega,
\end{aligned}
$$

where $\omega$ is as usual the vacuum mixing angle defined earlier. In the three flavour case the only relevant probability is,

$$
P_{e e}=\left(1-\epsilon^{2}\right)\left[\left(1-P_{L}\right) \sin ^{2} \omega+P_{L} \cos ^{2} \omega\right] .
$$

Note that, since $\epsilon$ is small, the flux at the detector is entirely controlled by $\omega$ and $P_{L}$ which is also a function of $\omega$.

\section{B. Matter effects for antineutrinos}

We now consider the case of $\bar{\nu}_{e}$ propagation in highly dense matter. The only change in this case is that the matter dependent term in the relevant part of the mass squared matrix has the opposite sign (to that in Eq. (17)), that is,

$$
A_{i}(r)=-\sqrt{2} G_{F} N_{i}(r) \times 2 E ; N_{1}=N_{e}, N_{2}=N_{n} / 2 .
$$

The analysis goes through as in the case of $\nu_{e}$ propagation through matter. There are no Landau-Zener jumps to consider in this case since the resonance conditions are never satisfied unless the mass hierarchy is altered. The propagation is therefore adiabatic and the survival probability is obtained by simply projecting the $\left|\bar{\nu}_{1}\right\rangle$ eigenstate on to the flavour eigenstate in vacuum (at the detector). The antineutrino survival and transition probabilities for $e$ and $s$ flavour neutrinos are,

$$
\begin{aligned}
& P_{\overline{e e}}=\left(1-2 \epsilon^{2}\right) \cos ^{2} \omega, \\
& P_{\overline{e s}}=\left(1-2 \epsilon^{2}\right) \sin ^{2} \omega, \\
& P_{\overline{s e}}=\left(1-2 \epsilon^{2}\right) \sin ^{2} \omega+2 \epsilon^{2} \sin 2 \omega, \\
& P_{\overline{s s}}=\left(1-2 \epsilon^{2}\right) \cos ^{2} \omega-2 \epsilon^{2} \sin 2 \omega,
\end{aligned}
$$

where $\omega$ is as usual the vacuum mixing angle defined earlier. As before, we do not need to know the other probabilities for our analysis.

\section{FLUXES AT THE DETECTOR}

We briefly compute the neutrino flux at the detector in the presence of mixing and compare the three and four flavour scenarios.

Following Kuo and Pantaleone [2], we denote the flux distribution, $\mathrm{d} \phi_{\alpha}^{0} / \mathrm{d} E$, of a neutrino (or antineutrino) of flavour $\alpha$ with energy $E$ produced in the core of the supernova by $F_{\alpha}^{0}$. In particular we use the generic label $F_{x}^{0}$ for flavours other than $\nu_{e}$ and $\bar{\nu}_{e}$ since

$$
F_{x}^{0}=F_{\mu}^{0}=F_{\bar{\mu}}^{0}=F_{\tau}^{0}=F_{\bar{\tau}}^{0} .
$$

All these flavours are produced via the neutral-current (NC) pair production processes and therefore have the same flux for all practical purposes. However, the $\nu_{e}$ and $\bar{\nu}_{e}$ fluxes are 
different from each other and the rest since they are produced not only by pair production but also derive contribution from charged-current (CC) processes. Note that there is no production of sterile neutrinos at source.

The flux reaching the detector from a supernova at a distance $d$ from earth is reduced by an overall geometric factor of $1 /\left(4 \pi d^{2}\right)$. Apart from this, there is a further modification of the observed flux due to oscillations in the presence of matter. The flux on earth, in the various flavours, is given in terms of the flux of neutrinos produced in the core of the supernova by,

$$
\begin{aligned}
F_{e} & =P_{e e} F_{e}^{0}+P_{e \mu} F_{\mu}^{0}+P_{e \tau} F_{\tau}^{0}, \\
& =P_{e e} F_{e}^{0}+\left(1-P_{e e}-P_{e s}\right) F_{x}^{0},
\end{aligned}
$$

where we have made use of the constraint $\sum_{\beta} P_{\alpha \beta}=1$. Since $\nu_{\mu}$ and $\nu_{\tau}$ induced events cannot be separated in water Cerenkov detectors, their combined flux on earth may be written as

$$
\begin{aligned}
2 F_{x} & =F_{\mu}+F_{\tau}, \\
& =\left(P_{e e}+P_{e s}+P_{s e}+P_{s s}\right) F_{x}^{0}+\left(1-P_{e e}-P_{s e}\right) F_{e}^{0},
\end{aligned}
$$

and

$$
F_{s}=\left(1-P_{s s}-P_{s e}\right) F_{x}^{0}+P_{s e} F_{e}^{0}
$$

Note that a part of active neutrino flavours are converted into sterile due to mixing. The total flux is however conserved since,

$$
F_{e}+2 F_{x}+F_{s}=F_{e}^{0}+2 F_{x}^{0}
$$

For comparison, the three-flavour case may be obtained by setting all the conversion probabilities involving the sterile neutrino to zero, and setting $P_{s s}=1$.

\section{ANALYTICAL RESULTS FOR FLUXES}

Without any numerical estimation, or choice of supernova model, it is possible to analyse the adiabatic case as well as the extreme nonadiabatic case and obtain gross features of the mixing for both 3 and 4 flavour mixings. We shall analyse the mixings in the neutrinos and antineutrinos separately. We begin with the adiabatic case.

\section{A. Neutrino fluxes: Adiabatic case}

For adiabatic propagation, Landau Zener jumps must be negligible. This in turn means that the larger the mass difference, the smaller the mixing angle one can accomodate. We shall assume that this is satisfied for the case under consideration here.

The results for the neutrino fluxes are given in Table $\mathbb{1}$. It is seen that the fluxes are independent of the (12) mixing angle, $\omega$, in all cases. 


\section{4-flavours}

For the 4-flavour case, it is striking that the electron neutrino flux and the sterile neutrino flux (not observable in the detector) are equal and completely deplete the original spectrum in $\nu_{\mu, \tau}$. The $\nu_{\mu, \tau}$ flux at the detector is entirely made up by almost complete conversion of electron neutrinos. From the supernova models, it is reasonably well known that the spectrum of $\nu_{e}$ is colder than $\nu_{\mu, \tau}$. In effect therefore the mixing interchanges the hot and the cold spectra in the supernova, with about half of the hotter spectrum lost in the sterile neutrinos.

\section{3-flavours}

The 3-flavour $\nu_{e}$ flux is not very different from the 4-flavour one: about half of the hotter spectrum is lost and reappears as the electron neutrino spectrum in the detector (see Table [). There is no sterile neutrino; hence the $\nu_{\mu, \tau}$ spectra are not further depleted, in contrast to the 4-flavour case. However, this difference in $\nu_{\mu, \tau}$ spectra between 3 and 4 flavour mixing is not easily observed since water Cerenkov detectors see very few events from flavours other than electron type neutrinos. The evidence for physical loss of flux into a sterile channel may be observed from neutral current events in detectors such as SNO.

In both 3 and 4 flavour cases, there is mixing into the $\nu_{e}$ spectrum of the hotter $\nu_{x}$ spectrum. As we have seen in I, this admixture of hotter spectrum is signalled by a strong increase in the number of backwardly peaked events due to the opening up of the $\nu O$ charged current channel in the detector, and should be a clean indicator of $\nu_{e}-\nu_{x}$ mixing.

\section{B. Antineutrino fluxes}

The basic analysis is similar to the case of neutrino fluxes given in equations (21), (22) and (23). The changes occur only at the level of substituting for the appropriate probabilities, given in the previous section. These are different for neutrinos and antineutrinos.

Unlike in the neutrino sector, predictions in the anti-neutrino sector require inputs on $\omega$. Within the schemes considered in this paper, this is obtained only from an analysis of the solar neutrino deficit. Then there are two possible solutions for $\sin \omega$ - the so-called small mixing angle (SMA) MSW solution, and also the large mixing angle (LMA) solutions. The LMA solutions arise both with matter effects included and in the so-called just-so solutions where there are no matter effects. At present, all three scenarios are consistent with the solar neutrino data although the mass-squared difference required in these cases is vastly different- $\delta m_{12}^{2} \approx 10^{-5} \mathrm{eV}^{2}$ in the case of MSW solution with LMA, $\delta m_{12}^{2} \approx 10^{-7} \mathrm{eV}^{2}$ for LMA with vacuum (LMA-V) and $\delta m_{12}^{2} \approx 10^{-6} \mathrm{eV}^{2}$ for SMA solution.

In any case, whatever the value of $\omega$, the propagation of antineutrinos always remains adiabatic (unless we choose an inverted mass hierarchy, which we do not consider here). The results for arbitrary $\omega$ are shown in Table II]. While there is hardly any admixture of the hotter $\bar{\nu}_{\mu, \tau}$ spectrum into $\bar{\nu}_{e}$ (independent of $\omega$ ) in the 4-flavour case, the extent of this

mixing depends on $\omega$ in the 3-flavour case. 


\section{Small $\omega$}

It is easy to see from Table III that for small $\omega$, the $\bar{\nu}_{e}$ flux is almost unaltered, in both 3 and 4-flavour mixing cases. These are then indistinguishable (and also indistinguishable from the no-mixing case).

\section{Large $\omega$}

In 4-flavour mixing, the original $\bar{\nu}_{e}$ flux is depleted by a factor of $\cos ^{2} \omega$, while still receiving no contribution from $\nu_{\mu, \tau}$ (See Table [II). This depletion is seen at all energies and should be easily observable since the CC process $\bar{\nu}_{e} p \rightarrow e^{+} n$ has by far the largest cross-section in the detector; furthermore, it is approximately isotropic in distribution [10]. In contrast, a large value of $\omega$ increases the contribution of $\nu_{\mu, \tau}$ in $\bar{\nu}_{e}$ while proportionately decreasing the survival of the original $\bar{\nu}_{e}$ in 3 flavours. Such a mixing will be observable at the higher energy end of the spectrum, where, however, the event rates are low. Hence, the behaviour of the isotropic events will be a clean signal of the number of flavours, provided, of course, that the original fluxes are accurately known. This depends on the models of stellar collapse. However, it may be possible to normalise the overall spectrum from the initial burst neutrinos (which are purely $\nu_{e}$ type and give a forward electron distribution in the Cerenkov detector).

The $\bar{\nu}_{x}$ flux is essentially unaltered, independent of $\omega$ in the case of 4 flavours. With 3 flavours, the original $\bar{\nu}_{x}$ flux is depleted, and is compensated for by a proportional contribution from the $\bar{\nu}_{e}$ flux. Hence the results are reversed compared to the $\bar{\nu}_{e}$ case. This signal, however, may be difficult to observe as there are very few events in this channel.

\section{Sterile neutrino fluxes}

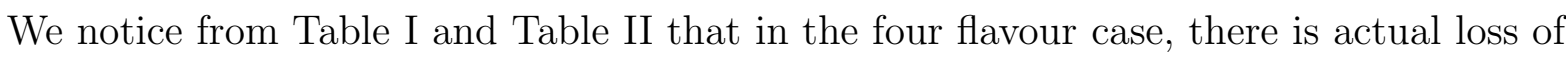
flux into the sterile channel. Independent of $\omega$, half of the hot $\nu_{\mu, \tau}$ spectrum is lost into the $\nu_{s}$ channel, while a portion proportional to $\sin ^{2} \omega$ of the $\bar{\nu}_{e}$ flux is lost into the $\bar{\nu}_{s}$ channel. From a detection point of view, this change, even if dramatically large, cannot be observed, unless through observation of neutral current events.

\section{Neutrino fluxes: Non-adiabatic case}

As we have seen, the various survival and transition probabilities in the non-adiabatic case depend on both $\epsilon$ and $\omega$. For a large range of $\epsilon$ the propagation is adiabatic. Hence non-adiabatic effects are important only for small values of $\epsilon$ when the jump probability at the upper resonances abruptly changes to one. Then, the flux in this case is driven entirely by $\omega$, which also determines the size of the Landau-Zener jumps at the lower resonance through the value of the jump probability, $P_{L}$. 


\section{Small $\omega$}

The neutrino fluxes at the detector, calculated using these probabilities, are summarised in Table [II. For comparison we have also given the results for three flavours. It is clear from the table that if the mixing angle $\omega$ is small, the $\nu_{e}$ flux at the detector is approximately given by,

$$
\begin{aligned}
& F_{e} \approx P_{L} F_{e}^{0}+\left(1-P_{L}\right) F_{x}^{0},(3 \text {-flavours }), \\
& F_{e} \approx P_{L} F_{e}^{0} \quad \text { (4-flavours) } .
\end{aligned}
$$

Hence the signals in the 3 and 4 flavour cases are drastically different: there is no mixing of the hot spectrum into the original $\nu_{e}$ spectrum in the 4 flavour case.

\section{Large $\omega$}

In the case of maximal mixing, $\omega=\pi / 4$, we have

$$
\begin{aligned}
& F_{e} \approx \frac{1}{2}\left[F_{e}^{0}+F_{x}^{0}\right],(3 \text {-flavours) } \\
& F_{e} \approx \frac{1}{2} F_{e}^{0} \quad \text { (4-flavours) } .
\end{aligned}
$$

Near maximal mixing, the LZ jump probability $P_{L}$ plays no role at all. However, the $\nu_{e}$ flux at the detector again differs dramatically from three to four flavours (See Table IIII). The three flavour result is also unlike the fully adiabatic case since we have an equal mixture of the cold and the hot spectrum due to mixing. Furthermore, the four flavour scenario differs from the four flavour adiabatic case quite dramatically. Unlike the adiabatic case, here there is no contribution from the hot spectrum, while the cold spectrum is depleted by about half.

In short, the $\nu_{e}$ signal in the nonadiabatic case is very different not only between 3 and 4 flavours, but also is very different from the adiabatic case. Here we have discussed the two extreme cases where the lower resonance undergoes or does not undergo Landau Zener jumps. We clarify the general case numerically below.

In the case of anti-neutrinos, non-adiabatic transitions do not occur since there are no level crossings with the mass hierarchy assumed in this analysis. However, in the case of an inverted mass hierarchy that allows for non-adiabatic proagation in the antineutrino sector, the survival probabilities are identical to those given above. We will not discuss this situation further here.

In the next section, we compute numerically the fluxes, for some preferred values of $\omega$ and $\delta m_{12}^{2}$ (obtained from an analysis of solar neutrino data) with the jump probability determined by these parameters. We will also predict event rates and the total number of events in the different cases we have discussed above.

\section{CROSS-SECTIONS AND EVENT RATES}

The basic quantity we are interested in is the distribution of events in the detector as a function of the energy of the detected particle. For a detailed discussion, for example, see I. Since we are interested in water Cerenkov detectors, the relevant interactions are, 


$$
\begin{aligned}
\nu_{\ell}\left(\bar{\nu}_{\ell}\right)+e^{-} & \rightarrow \nu_{\ell}\left(\bar{\nu}_{\ell}\right)+e^{-}, \ell=e, \mu, \tau \\
\bar{\nu}_{e}+p & \rightarrow e^{+}+n \\
\nu_{e}+{ }^{16} O & \rightarrow e^{-}+{ }^{16} F \\
\bar{\nu}_{e}+{ }^{16} O & \rightarrow e^{+}+{ }^{16} N .
\end{aligned}
$$

The oxygen cross-sections have been taken from Fig. 1 of Haxton [3]. All other cross-sections are well known. As the interactions on protons and oxygen nuclei are purely CC interactions, they involve only $\nu_{e}$ and $\bar{\nu}_{e}$. The interaction with electrons involves both $\mathrm{CC}$ and $\mathrm{NC}$ interactions for $\nu_{e}$ and $\bar{\nu}_{e}$ and only NC interactions for all other flavours. The $\bar{\nu}_{e} p$ crosssection is the largest, being proportional to the square of the antineutrino energy. In terms of total number of events, therefore, water Cerenkov detectors are mostly dominated by $\bar{\nu}_{e}$ events. However, the different interactions in the detector have distinct angular distributions: The elastic neutrino-electron cross-sections are forward peaked, especially for neutrinos with energies $\gtrsim 10 \mathrm{MeV}$ 15, 16, while the proton cross-section is approximately isotropic in the lab frame. There is a slight excess of backward events for energies below $15 \mathrm{MeV}$, and slight excess in the forward direction at higher energies [10] but the excess in either direction at the relevant energies are limited to few percent of the total number of events. Finally, the CC $\nu_{e}\left(\bar{\nu}_{e}\right)$ cross-section on oxygen, although having a rather large threshold of $15.4 \mathrm{MeV}$ $(11.4 \mathrm{MeV})$ [3], increases rapidly with incoming neutrino energy and the number of events in the backward direction increases substantially with energy of the incoming neutrino above the threshold.

The time integrated event rate, from neutrinos of flavour $\alpha$ and energy $E$, as a function of the recoil electron (or positron) energy, $E_{e}$, is as usual given by,

$$
\frac{\mathrm{d} N_{\alpha}^{t, p}\left(E_{e}\right)}{\mathrm{d} E_{e}}=\frac{n_{t}}{4 \pi d^{2}} \sum_{b} \Delta t_{b} \int \mathrm{d} E F_{\alpha}(b) \frac{\mathrm{d} \sigma_{p}}{\mathrm{~d} E_{e}} .
$$

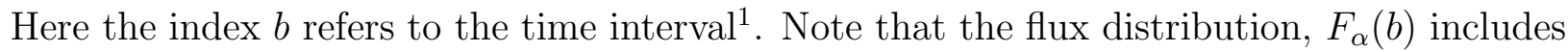
the effects of mixing in the hot dense core and is a function of the time-dependent temperature, $T_{b}$. The index $p$ refers to any of the various processes through which the neutrino $\alpha$ can interact with the target, $t$, in the detector. Here $n_{t}$ refers to the number of scattering targets (of either $e, p$ or ${ }^{16} O$ ) that are available in the detector. The total number of events from a given flavour of neutrino in a given bin, $k$, of electron energy (which we choose to be of width $1 \mathrm{MeV}$ ) then is

$$
N_{\alpha}^{t, p}(k)=\int_{k}^{k+1} \mathrm{~d} E_{e} \frac{\mathrm{d} N_{\alpha}^{t, p}}{\mathrm{~d} E_{e}} .
$$

In the next section we give detailed numerical estimates both for the spectrum and the integrated events for some specific representative values of $\epsilon$ and $\omega$, as constrained by other experiments.

\footnotetext{
${ }^{1}$ The constant factor $\Delta t_{b}$ should also appear in the corresponding equation, Eq. (27), in I.
} 


\section{RESULTS AND DISCUSSION}

As in I, we compute the time integrated event rate at a prototype 1 Kton water Cerenkov detector from neutrinos emitted by a supernova exploding $10 \mathrm{KPc}$ away. Results for any other supernova explosion may be obtained by scaling the event rate by the appropriate distance to the supernova and the size of the detector, as shown in Eq. (31). We assume the efficiency and resolution of such a detector to be perfect.

We use the luminosity and average energy distributions (as functions of time) as given in Totani et al. [18], based on the numerical modelling of Mayle, Wilson and Schramm [19]. In a short time interval, $\Delta t_{b}$, the temperature can be set to a constant, $T_{b}$. Then, the neutrino number flux is described, in this time interval, by a thermal Fermi Dirac distribution,

$$
F_{\alpha}^{0}(b)=N_{0} \frac{\mathcal{L}(\alpha)}{T_{b}^{4}} \frac{E^{2}}{\left(\exp \left(E / T_{b}\right)+1\right)},
$$

for neutrinos of flavour $\alpha$ and energy $E$ at a time $t$ after the core bounce. Here $b$ refers to the time-bin, $t=t_{0}+b \Delta t$. Hereafter, we set the time of bounce, $t_{0}=0$. The overall normalisation, $N_{0}$, is fixed by requiring that the total energy emitted per unit time equals the luminosity, $\mathcal{L}(\alpha)$, in that time interval. The total emitted energy in all flavours of neutrinos is about $2.7 \times 10^{53} \mathrm{ergs}$, which is more or less equally distributed in all flavours. The number of neutrinos emitted in each flavour, however, is not the same since their average energies are different.

It turns out that the results are not very sensitive to the time dependence of the temperature profile. For instance, using time-independent temperatures of 11, 16, and $25 \mathrm{MeV}$ for the $\nu_{e}, \bar{\nu}_{e}$ and the $\nu_{x}$ thermal spectra (instead of the above time-dependent ones) changes the results by less than $5 \%$ in the antineutrino sector and by about $8 \%$ in the neutrino sector. However the difference between the average temperatures of the electron and other types of neutrinos is crucial to the analysis.

In addition we impose the following known constraints on the mixing matrix in vacuum both for three and four flavour scenarios. Consistent with the CHOOZ constraint, namely $\epsilon \leq 0.16$ (which we have already imposed at the level of the parametrisation itself), we set $\epsilon=0.087$. Furthermore, the following constraints derived from solar and atmospheric neutrino observations are also taken into consideration:

The constraint from the atmospheric neutrino analysis implies that the relevant angle $\psi(\approx \pi / 4)$, is near maximal and the relevant mass squared difference is of the order of $10^{-3}$. Neither of these constraints directly enter our calculations except to determine whether the upper resonance is adiabatic or not depending on the value of $\epsilon$ as constrained by the CHOOZ findings. We consider both here.

The more relevant constraint follows from the solar neutrino physics. Here there are three possible best fits to the combined data on solar neutrinos [17]:

1. $\sin ^{2}(2 \omega)=6.0 \times 10^{-3}, \delta m_{12}^{2}=5.4 \times 10^{-6} \mathrm{eV}^{2}(\mathrm{SMA})$. The small angle MSW solution.

2. $\sin ^{2}(2 \omega)=0.76, \delta m_{12}^{2}=1.8 \times 10^{-5} \mathrm{eV}^{2}$ (LMA). The large angle MSW solution.

3. $\sin ^{2}(2 \omega)=0.96, \delta m_{12}^{2}=7.9 \times 10^{-8} \mathrm{eV}^{2}(\mathrm{LMA}-\mathrm{V})$. The large angle vacuum solution. 
While choosing only these values may appear restrictive, it will be seen that these values cover the typical ranges within which all the allowed changes take place. The numerical calculations are done by following the evolution of the mass eigenstates through all the resonances including the appropriate jump probabilities.

We will now discuss the results for all these choices. We choose $\epsilon=0.087$ for the adiabatic case and $\epsilon \sim 0$ for the nonadiabatic one. Whether or not the Landau Zener jumps play a

role is determined by the value of $\omega$ we choose. The interaction at the detector is mainly of three types:

1. Isotropic events: These are by far the largest fraction of the events, and are due to $\bar{\nu}_{e} p \rightarrow e^{+} n$.

2. Forward peaked events: These are due to elastic scatterings of $\nu_{e}, \bar{\nu}_{e}, \nu_{x}, \bar{\nu}_{x}$ on electron targets.

3. Backward peaked events: These arise from CC scattering of $\nu_{e}$ and $\bar{\nu}_{e}$ on oxygen nuclei in the target.

Hence, angular information on the final state electron $\left(e^{-}\right.$or $\left.e^{+}\right)$will allow us to separate out the above three types of events. Note however that the extent of backward peaking is severely dependent on the spectral temperature [3]. For the temperatures corresponding to the $\nu_{x}$ spectrum, we can assume the events to be totally in the backward direction.

\section{A. Total Number of events}

We give the time integrated number of events of the scattered electron, with energy, $E_{e}>8 \mathrm{MeV}$ (which is a typical threshhold for such detectors), in Tables 4 to 6, for the three choices of $\omega$. These are the number of events for a supernova explosion at $10 \mathrm{Kpc}$ for a 1 kTon water Cerenkov detector.

The first column in all the tables corresponds to the base case of no mixing. The next two columns correspond to the adiabatic result $(\epsilon=0.087$ for the three and four flavour case, while the last two columns correspond to the case where $\epsilon \sim 0$. The isotropic events (due to $\nu_{e}$ ) are insensitive to values of $\epsilon$ chosen. The first table corresponds to small $\omega$, where the lower resonance can involve Landau Zener jumps, while the next two correspond to large $\omega$ and are adiabatic at the lower resonance.

It is expected that the integrated events are less sensitive to the details of models of stellar collapse, especially the energy distribution of the fluxes. Hence the predictions are more stable.

$$
\text { 1. } \epsilon=0.087 \text { (Adiabatic case) }
$$

The large backward excess due to mixing of a hot spectrum into the $\bar{\nu}_{e}$ spectrum is a clear signal of mixing, irrespective of the number of flavours involved, as seen in Tables 4-6. Variations in the forward events may not be visible. However, the large depletion in the isotropic events with increasing $\omega$ (in the 4 flavour case) can distinguish between the 3 and 4 flavour mixing, especially when $\omega$ is large (see Tables 5 and 6). 
Here the lower resonance may be adiabatic or nonadiabatic, depending on the value of $\omega$. Again here a large excess of backward events clearly indicates the presence of mixing, but this is seen only for the 3 flavour case (see Tables 4-6). The isotropic background from the $\bar{\nu}_{e} p$ scattering is again sensitive to mixing and the number of mixing flavours, for large $\omega$ (tables 5 and 6 ). Finally, unlike the previous case the depletion (enhancement) of forward events, independent of $\omega$, for 4(3) flavours may be observable, especially in the differential event rates, $\mathrm{d} N / \mathrm{d} E_{e}$. It is therefore interesting to study the differential spectrum, which obviously has more information than the integrated events, to see whether this can be quantified further. We do this below.

\section{B. Event rates}

The event rates as a function of the scattered electron energy, $E_{e}$, are displayed in Figs. 3, 4, and 5. For detailed results in the 3-flavour case, see I. The solid lines in all the figures refer to the case when there is no mixing and serve as a reference. Both the three- and four-flavour results are displayed in each of these figures.

In Fig. 3 we show the time integrated event rate per unit electron energy bin (of $1 \mathrm{MeV}$ ) as a function of the detected electron energy for the adiabatic case when $\epsilon=0.087$. The dashed curves correspond to the case with mixing. Interactions with both electrons as well as oxygen nuclei occur. The event rates in both cases are shown. In both cases, mixing progressively enhances the high $E_{e}$ event rates. This shows up as an increase in both the forward and abckward peaked events. There is no perceptible difference between the 3- and 4-flavour scenarios, independent of constraints on the angle $\omega$.

In Fig. 4 we show the electron neutrino spectrum for the case when $\epsilon$ is small, in fact near zero. Hence the propagation near the upper resonances is nonadiabatic. Here, 3 and 4 flavour mixing give drastically different results. For all $\omega$, the forward and backward rates are depleted for 4 flavour mixing. However, these rates are small. The 3 flavour nonadiabatic mixing is indistinguishable from the adiabatic case (compare the left hand sides of Figs. 3 and 4).

Thus, an enhancement in the backward peaked events clearly indicates the presence of mixing. But there is no unambigious indicator of the number of flavours unless the parameters get restricted better through solar neutrino solutions. For this, we need to examine the electron anti-neutrino spectrum.

In Fig. 5 we show the $\bar{\nu}_{e}$ spectrum as a function of energy for three different choices of $\omega$. (Here $\epsilon=0.087$ but the results are essentially the same even if $\epsilon$ is nearly zero.) The propagation is always adiabatic for the antineutrinos since there are no resonances to account for nonadiabatic jumps. The $\bar{\nu}_{e}$ can interact with electrons, oxygen and protons in water and therefore contribute to forward, backward and isotropic events, the most dominant one being the approximately isotropic events due to interaction on the proton target (this cross-section is about two orders of magnitude larger than the others, for typical energies involved). For the SMA solution, there is no perceptible difference between the three and four flavour scenarios and the no mixing case, as already discussed. A perceptible difference occurs for LMA and LMA-V solutions. The intermediate energy $\left(E_{e} \sim 20 \mathrm{MeV}\right)$ spectrum 
is not depleted for 3 flavour mixing, while it is depleted for 4 flavour mixing (for the LMA solutions). Again, the 3 flavour isotropic spectrum is enhanced at larger energies (See Fig. 5); no such enhancement occurs in 4 flavours. Such an energy dependent pattern of event depletion and enhancement may help distinguish not only the 3 from the 4 flavour case, but also the mixing from the no-mixing solution. This pattern will survive, independent of uncertainties in the overall flux normalisations; however, it must be remembered that the event rate is very small at large energies $\left(E_{e}>40 \mathrm{MeV}\right)$.

It is therefore seen that a combination of detection of both isotropic and forward events at low and intermediate energies (involving both $\bar{\nu}_{e} p$ and $\nu_{e} e$ scattering in water) can help distinguish the 3 and 4 flavour solutions for the case when $\epsilon$ is close to zero.

\section{CONCLUSIONS}

To summarise, neutrino mixing in the 3- and 4- flavour cases gives rise to very different event rates due to neutrinos (and antineutrinos) from stellar collapse interacting with a water Cerenkov detector. The neutrino sector is sensitive to the presence of mixing. This shows up typically as an enhancement of the higher energy events in the backward direction. This signal is not sensitive to the number of flavours. For a fairly large region of parameter space, the antineutrino events are sensitive to the number of flavours involved. Since this is the main signal of stellar collapse, the statistics should be good enough to make rather strong predictions from this channel.

We would like to thank the organisers of the 6th Workshop on High Energy Physics Phenomenology (WHEPP-6), Chennai, January 2000, where a part of this work was done. We thank Sandhya Choubey, Srubabati Goswami, Kamales Kar, and Debashis Majumdar for discussions during this meeting. We would also like to thank Hari Dass for discussions.

\section{APPENDIX A}

We clarify here our choice of mixing matrix that we have used in the text. This arises from the requirement of consistency with various experiments. We discuss this, for the case of vacuum mixing, for both three and four flavour solutions. We shall set all CP violating phases to zero in what follows.

For the sake of completeness, we briefly quote the results for the three flavour case, where all flavours are active neutrinos. We choose the mixing angles to be $\omega, \phi$ and $\psi$ for the (12), (13) and (23) mixings. The electron neutrino survival probability is given by,

$$
\begin{aligned}
P_{e e}= & \left(1-2 c_{\phi}^{2} s_{\phi}^{2}\right)-2 c_{\phi}^{4} c_{\omega}^{2} s_{\omega}^{2}+2 c_{\phi}^{4} c_{\omega}^{2} s_{\omega}^{2} \cos \left(2.54 \delta_{12} L / E\right) \\
& +2 c_{\phi}^{4} s_{\phi}^{2}\left[c_{\omega}^{2} \cos \left(2.54 \delta_{13} L / E\right)+s_{\omega}^{2} \cos \left(2.54 \delta_{23} L / E\right)\right],
\end{aligned}
$$

where $L$ is measured in meters, $\delta$ in $\mathrm{eV}^{2}$ and $E$ in $\mathrm{MeV}$.

We first assume a set of weak constraints, viz., that there exist two different scales for $\delta: \delta_{12} \leq 10^{-5} \mathrm{eV}^{2}$ from solar neutrino data, and $\delta_{13} \sim 10^{-3} \mathrm{eV}^{2}$ from atmospheric neutrino data. Hence $\delta_{13} \simeq \delta_{23}=\delta$. 
For the case of CHOOZ, we have $L / E \sim 300$ [12]. Then the electron neutrino survival probability that can be applied in the $\mathrm{CHOOz}$ experiment reduces to

$$
P_{e e}=1-4 \epsilon^{2} \sin ^{2}\left(1.27 \frac{\delta L}{E}\right),
$$

independent of $\omega$, where we have set $\sin \phi=\epsilon$. Hence the CHOOz result constrains $\epsilon$ to be small; in particular, $\epsilon<0.16$, which justifies the approximation used in our parametrisation of the three-flavour mixing matrix. As a result one cannot accomodate the LSND results [11] in a three-flavour frame-work.

Hence it is necessary, for the sake of consistency of atmospheric neutrinos, CHOOz and LSND, to have at least one extra (sterile) flavour.

We now discuss the four-flavour scenario within the mass hierarchy shown in Figure 1. The same weak constraints as in the three-flavour case are assumed here. This results in a two-doublet structure for the four levels where the doublets are separated by the mass scale imposed by LSND, namely, $\delta_{13} \simeq \delta_{14} \simeq \delta_{23} \simeq \delta_{24} \sim 0.1-1 \mathrm{eV}^{2}$. In addition to these mass squared differences, there are now six mixing angles, $\theta_{12}=\omega, \theta_{34}=\psi, \theta_{13}=\theta_{1}, \theta_{14}=\theta_{2}$, $\theta_{23}=\theta_{3}, \theta_{24}=\theta_{4}$, where $\psi$ is constrained to be maximal, $\psi=\pi / 4$, from atmospheric neutrino data. Hence the mixing matrix is

$$
U=U_{\psi} U_{4} U_{3} U_{2} U_{1} U_{\omega} .
$$

We first discuss the constraint on $P_{e e}$ from $\mathrm{CHOOz}$ in the 4-flavour case. Because $L / E$ is large, of the order of 300 , the oscillatory term involving $\delta_{13}$ averages out to zero:

$$
\left\langle\cos \left(\frac{\delta_{13} L}{2 E}\right)\right\rangle=0 \text {. }
$$

The same holds for other mass squared differences of the same order of magnitude as $\delta_{13}$. Furthermore, since $\delta_{12}$ is small, we we can set $\cos \left(\delta_{12} L /(2 E)\right) \sim 1$. Therefore the survival probability of the electron neutrino is,

$$
P_{e e}=1-\frac{1}{2} \sin ^{2} 2 \theta_{2}-\frac{1}{2} \cos ^{4} \theta_{2} \sin ^{2} 2 \theta_{1}+\frac{1}{2} \sin ^{2} 2 \theta_{2} \sin ^{2} 2 \theta_{1} \cos \left(\frac{\delta_{34} L}{2 E}\right),
$$

where the scale $\delta_{34}$ is set by the atmospheric neutrino data so that the oscillatory term is of order one. Note that the survival probability does not involve $\theta_{3}$ and $\theta_{4}$, as remarked in the text.

Then the CHOOZ result, $P_{e e}=1 \pm 0.04$ [12] indicates that both $\theta_{1}$ and $\theta_{2}$ should be small. We have therefore chosen these to be of the same order: $\sin \theta_{1} \sim \sin \theta_{2} \sim \epsilon$. Then

$$
\begin{aligned}
P_{e e} & =1-4 \epsilon^{2}+2 \epsilon^{4} \cos \left(\frac{\delta_{34} L}{2 E}\right), \\
& \simeq 1-4 \epsilon^{2},
\end{aligned}
$$

independent of $\delta_{34}, L$, and $E$. The CHOOZ null result therefore provides an upper bound on these mixing angles.

Using all these constraints, the relevant transition probability for LSND is given by

$$
P_{\mu e}=2 \epsilon^{2}\left[\cos \theta_{3}-\sin \theta_{4} \sin \theta_{3}+\cos \theta_{4}\right]^{2} \sin ^{2}\left(\frac{\delta L}{4 E}\right) .
$$


Here $\delta$ is the separation between the two doublets.

The LSND result gives a non-zero value for $P_{\mu e}$, viz., $P_{\mu e}=(2.6 \pm 1.1) \times 10^{-3}$ [11]. This unambiguously fixes $\epsilon$ to be different from zero even though the CHOOZ result allows this value. Combining the limits from the two experiments, we have the constraint,

$$
\epsilon_{\mathrm{LSND}} \leq \epsilon \leq \epsilon_{\mathrm{CHOOZ}}
$$

The values we get in the four-flavour case are a completely consistent set. We have used allowed values of the various parameters from the above in the analysis of supernova neutrinos in the text.

Note that there are still no constraints on $\theta_{3}$ and $\theta_{4}$ which are the (23) and (24) mixing angles. Although we have set them to $\epsilon$ in the text, the electron (and anti-electron) survival probabilities that we define are independent of these mixing angles (since they do not involve electron-type transitions). Hence this choice does not affect the results that we have obtained for these probabilities. Furthermore, we remark that the present generation of experiments is unlikely to constrain these angles significantly. A more detailed analysis of the allowed parameter space in the case of four-flavour mixing will be published elsewhere. 


\section{REFERENCES}

[1] Gautam Dutta, D. Indumathi, M.V.N. Murthy and G. Rajasekaran, Phys. Rev. D61, 13009 (2000).

[2] T. K. Kuo and J. Pantaleone, Phys. Rev. D37, 298 (1988).

[3] W. C. Haxton, Phys. Rev. D36, 2283 (1987).

[4] J. F. Beacom and P. Vogel, Phys. Rev. D58, 053010 (1998); ibid 093012.

[5] Mohan Narayan, G. Rajasekaran, and S. Uma Sankar, Phys. Rev. D56, 437 (1997); Mohan Narayan, M. V. N. Murthy, G. Rajasekaran, and S. Uma Sankar, Phys. Rev. D53, 2809 (1996); G. L. Fogli, E. Lisi, A. Marrone, and G. Scioscia, Phys. Rev. D59, 117303 (1999); ibid 033001; G. L. Fogli, E. Lisi, and D. Montanino, Astropart. Phys. 9, 119 (1998); G. L. Fogli, E. Lisi, D. Montanino, and G. Scioscia, Phys. Rev. D56, 4365 (1997).

[6] Amol S.Dighe and Alexei Yu. Smirnov, hep-ph/9907423.

[7] Shao-Hsuan Chiu and T. K. Kuo, Phys. Rev. D61, 073015(2000)

[8] Sandhya Choubey and Kamales Kar, Preprint hep-ph/9905327.

[9] Petr Vogel, Invited talk at the 8th Int. Workshop on "Neutrino Telescopes", Venice Feb, 1999, astro-ph/9904338.

[10] J. F. Beacom, Invited talk at the 23rd Johns Hopkins Workshop on Current Problems in Particle Theory, Neutrinos in the next millennium, Baltimore, June 1999. hepph/9909231.

[11] C. Athanassopoulos, LSND Collaboration, Phys. Rev. Lett. 81, 1774 (1998); Phys. Rev. C, 2489 (1998).

[12] M. Apollonio et al., CHOOz Collab., Phys. Lett. B420, 397 (1998); Phys. Lett. B 466, 415 (1999) (hep-ex/9907037).

[13] L. Wolfenstein, Phys. Rev. D17, 2369 (1978); S. P. Mikheyev and A. Yu. Smirnov, Sov.J.Nucl.Phys, 42, 913 (1985); Nuovo Cimento, C9, 17(1986).

[14] S.J. Parke, Phys. Rev. Lett. 57; See also the review by T. K. Kuo and J. Pantaleone, Rev. Mod. Phys. 61, 937 (1989).

[15] J. N. Bahcall, Neutrino Astrophysics, Cambridge University Press, 1989.

[16] A. de Gouvêa and H. Murayama, Phys. Rev. Lett. 82, 3392 (1999).

[17] J.N. Bahcall, P.I. Krastev, and A.Yu. Smirnov, Phys. Rev. D 58, 96016 (1998).

[18] T. Totani, K. Sato, H.E. Dalhed, J. R. Wilson, Astrophys. J. 496, 216 (1998); see also the references in $[19$ below.

[19] A. Burrows and J.M. Lattimer, Astrophys. J. 307, 107 (1986); R. Mayle, J. R. Wilson and D. N. Schramm, Astrophys. J. 318, 288 (1987). 


\section{TABLES}

\begin{tabular}{|c|l|}
\hline \hline No. of flavours & Neutrino flux at detector, $F_{f}$ \\
\hline 3 & $F_{\nu_{e}}=\epsilon^{2} F_{\nu_{e}}^{0}+\left(1-\epsilon^{2}\right) F_{x}^{0}$ \\
4 & $F_{\nu_{e}}=\epsilon^{2} F_{\nu_{e}}^{0}+\left(1-2 \epsilon^{2}\right) F_{x}^{0}$ \\
\hline 3 & $2 F_{x}=\left(1+\epsilon^{2}\right) F_{x}^{0}+\left(1-\epsilon^{2}\right) F_{\nu_{e}}^{0}$ \\
4 & $2 F_{x}=\left(4 \epsilon^{2}\right) F_{x}^{0}+\left(1-2 \epsilon^{2}\right) F_{\nu_{e}}^{0}$ \\
\hline 4 & $F_{s}=\left(1-2 \epsilon^{2}\right) F_{x}^{0}+\left(\epsilon^{2}\right) F_{\nu_{e}}^{0}$ \\
\hline \hline
\end{tabular}

TABLE I. Neutrino fluxes observed at the detector in the extreme adiabatic limit.

\begin{tabular}{|c|l|}
\hline \hline \hline No. of flavours & Antineutrino flux at detector, $F_{f}$ \\
\hline 3 & $F_{\bar{e}}=\left(1-\epsilon^{2}\right) c_{\omega}^{2} F_{\bar{e}}^{0}+\left(s_{\omega}^{2}+\epsilon^{2} c_{\omega}^{2}\right) F_{x}^{0}$ \\
4 & $F_{\bar{e}}=\left(1-2 \epsilon^{2}\right) c_{\omega}^{2} F_{\bar{e}}^{0}+\left(2 \epsilon^{2}\right) F_{x}^{0}$ \\
\hline 3 & $2 F_{\bar{x}}=\left(1+c_{\omega}^{2}-\epsilon^{2} c_{\omega}^{2}\right) F_{x}^{0}+\left(s_{\omega}^{2}+\epsilon^{2} c_{\omega}^{2}\right) F_{\bar{e}}$ \\
4 & $2 F_{\bar{x}}=\left(2-4 \epsilon^{2}\right) F_{x}^{0}++2 \epsilon^{2}\left(1-s_{2 \omega}\right) F_{\bar{e}}$ \\
\hline 4 & $F_{\bar{s}}=2 \epsilon^{2} F_{x}^{0}+\left(s_{\omega}^{2}-2 \epsilon^{2}\left(s_{\omega}^{2}-s_{2 \omega}\right)\right) F_{\bar{e}}$ \\
\hline \hline
\end{tabular}

TABLE II. Antineutrino fluxes at the detector. $c_{\omega}=\cos \omega, s_{\omega}=\sin \omega$.

\begin{tabular}{|c|l|}
\hline \hline \hline No. of flavours & Neutrino flux at detector, $F_{f}$ \\
\hline 3 & $F_{e}=\left(1-\epsilon^{2}\right)\left[\left(1-P_{L}\right) s_{\omega}^{2}+P_{L} c_{\omega}^{2}\right] F_{e}^{0}$ \\
& $+\left[1-\left(1-\epsilon^{2}\right)\left(\left(1-P_{L}\right) s_{\omega}^{2}+P_{L} c_{\omega}^{2}\right)\right] F_{x}^{0}$ \\
4 & $F_{e}=\left(1-2 \epsilon^{2}\right)\left[\left(1-P_{L}\right) s_{\omega}^{2}+P_{L} c_{\omega}^{2}\right] F_{e}^{0}+2 \epsilon^{2} F_{x}^{0}$ \\
\hline 3 & $2 F_{x}=\left[1+\left(1-\epsilon^{2}\right)\left(\left(1-P_{L}\right) s_{\omega}^{2}+P_{L} c_{\omega}^{2}\right)\right] F_{x}^{0}$ \\
& $+\left[1-\left(1-\epsilon^{2}\right)\left(\left(1-P_{L}\right) s_{\omega}^{2}+P_{L} c_{\omega}^{2}\right)\right] F_{e}^{0}$ \\
4 & $2 F_{x}=2\left(1-2 \epsilon^{2}\right) F_{x}^{0}+2 \epsilon^{2}\left[1+\left(1-2 P_{L}\right) s(2 \omega)\right] F_{e}^{0}$ \\
\hline 4 & $F_{s}=2 \epsilon^{2} F_{x}^{0}+\left\{\left[\left(1-P_{L}\right) c_{\omega}^{2}+P_{L} s_{\omega}^{2}\right]-2 \epsilon^{2}\left[c_{\omega}^{2}-s_{\omega}^{2}-P_{L}\left[c_{2 \omega}+2 s_{2 \omega}\right]\right]\right\} F_{e}^{0}$ \\
\hline \hline
\end{tabular}

TABLE III. Neutrino fluxes at the detector when non-adiabatic effects are introduced. Note that while the transition is assumed to be fully non-adiabatic at the upper resonances, it is controlled by the probability $P_{L}$ at the lower resonance. 


\begin{tabular}{|r|r|r|r||r|r|}
\hline \hline Events & No Mixing & \multicolumn{2}{|c||}{ Mixing $(\epsilon=0.087)$} & \multicolumn{2}{c|}{ Mixing $(\epsilon \sim 0)$} \\
& & 3 flavour & 4 flavour & 3 flavour & 4 flavour \\
& & 7.6 & 6.9 & 7.1 & 4.4 \\
\hline Forward & 4.8 & 27.3 & 27.1 & 21.5 & 4.2 \\
\hline Backward & 281.1 & 282.0 & 282.2 & 281.3 & 280.7 \\
\hline Isotropic & \hline \hline
\end{tabular}

TABLE IV. Total number of events (for two different choices of $\epsilon$ ) in the forward and backward direction, and isotropic events, respectively, for a scattered electron energy above $8 \mathrm{MeV}$. Here $\sin ^{2}(2 \omega)=6 \times 10^{-3}, \delta^{2} m_{12}=5.4 \times 10^{-} 6 \mathrm{eV}^{2}$.

\begin{tabular}{|c|c|c|c|c|c|}
\hline \multirow[t]{2}{*}{ Events } & \multirow[t]{2}{*}{ No Mixing } & \multicolumn{2}{|c|}{$\operatorname{Mixing}(\epsilon=0.087)$} & \multicolumn{2}{|c|}{$\operatorname{Mixing}(\epsilon \sim 0)$} \\
\hline & & 3 flavour & 4 flavour & 3 flavour & 4 flavour \\
\hline Forward & 5.9 & 7.6 & 6.6 & 7.2 & 3.8 \\
\hline Backward & 4.8 & 29.5 & 26.2 & 23.8 & 3.1 \\
\hline Isotropic & 281.1 & 306.7 & 212.0 & 306.1 & 209.4 \\
\hline
\end{tabular}

TABLE V. Same as above for $\sin ^{2}(2 \omega)=0.76, \delta^{2} m_{12}=1.8 \times 10^{-} 5 \mathrm{eV}^{2}$.

\begin{tabular}{|r|r|r|r||r|r|}
\hline \hline Events & No Mixing & \multicolumn{2}{|c||}{ Mixing $(\epsilon=0.087)$} & \multicolumn{2}{c|}{ Mixing $(\epsilon \sim 0)$} \\
& & 3 flavour & 4 flavour & 3 flavour & 4 flavour \\
& & 7.7 & 6.5 & 7.0 & 4.0 \\
\hline Forward & 5.9 & 30.7 & 25.6 & 21.8 & 2.7 \\
\hline Backward & 4.8 & 320.8 & 171.9 & 320.3 & 168.7 \\
\hline Isotropic & 281.1 & & \\
\hline \hline
\end{tabular}

TABLE VI. Same as above for $\sin ^{2}(2 \omega)=0.96, \delta^{2} m_{12}=7.9 \times 10^{-} 8 \mathrm{eV}^{2}$. 


\section{FIGURES}

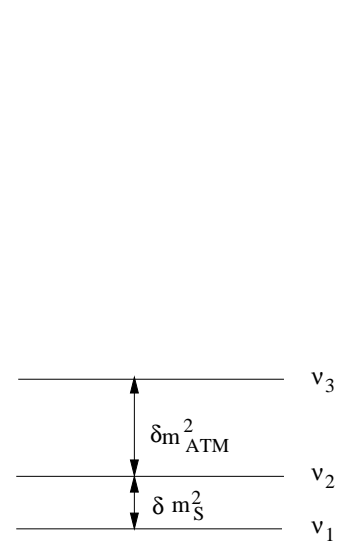

(a)

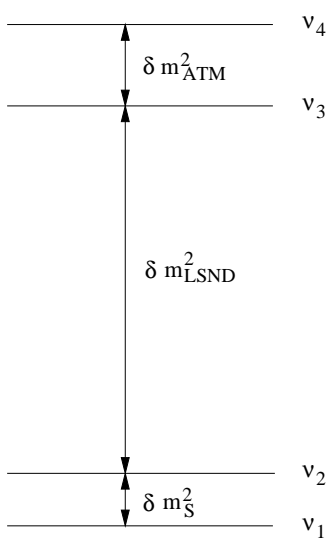

(b)

FIG. 1. The vacuum mass square differences in the 4 flavour schemes. Here $\nu_{e}$ and $\nu_{s}$ are predominantly mixed states of $\nu_{1}$ and $\nu_{2}$ while $\nu_{\mu}$ and $\nu_{\tau}$ are that of $\nu_{3}$ and $\nu_{4}$. The mixing between the lower and upper doublets has been chosen to be very small. Here S, ATM and LSND stand for the solar, atmospheric and LSND mass squared differences respectively. We have also shown the 3 flavour scheme for comparison.

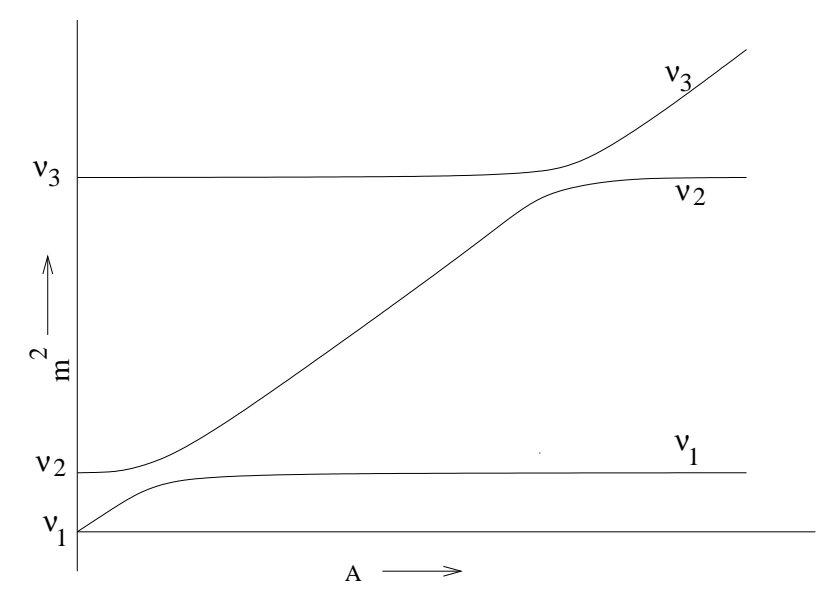

(a)

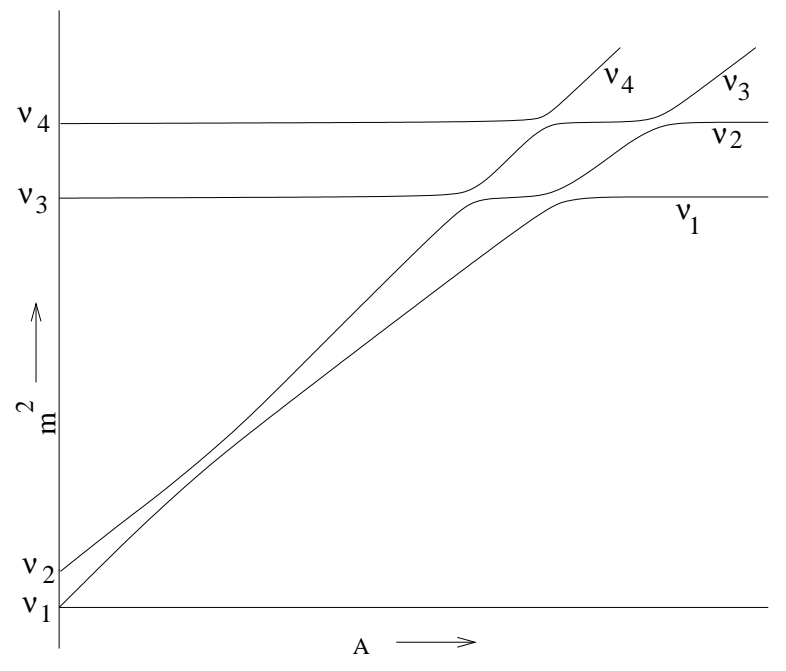

(b)

FIG. 2. Schematic drawing showing mass squares as functions of matter density in the 4 flavour scheme. Resonances occur at two different regions of matter density, the lower one at $A \approx \delta m_{S}^{2}$ and the upper one at $A \approx \delta m_{\mathrm{LSND}}^{2}$. The upper one consists of 4 close resonances. The three flavour scheme is also shown for comparison. 


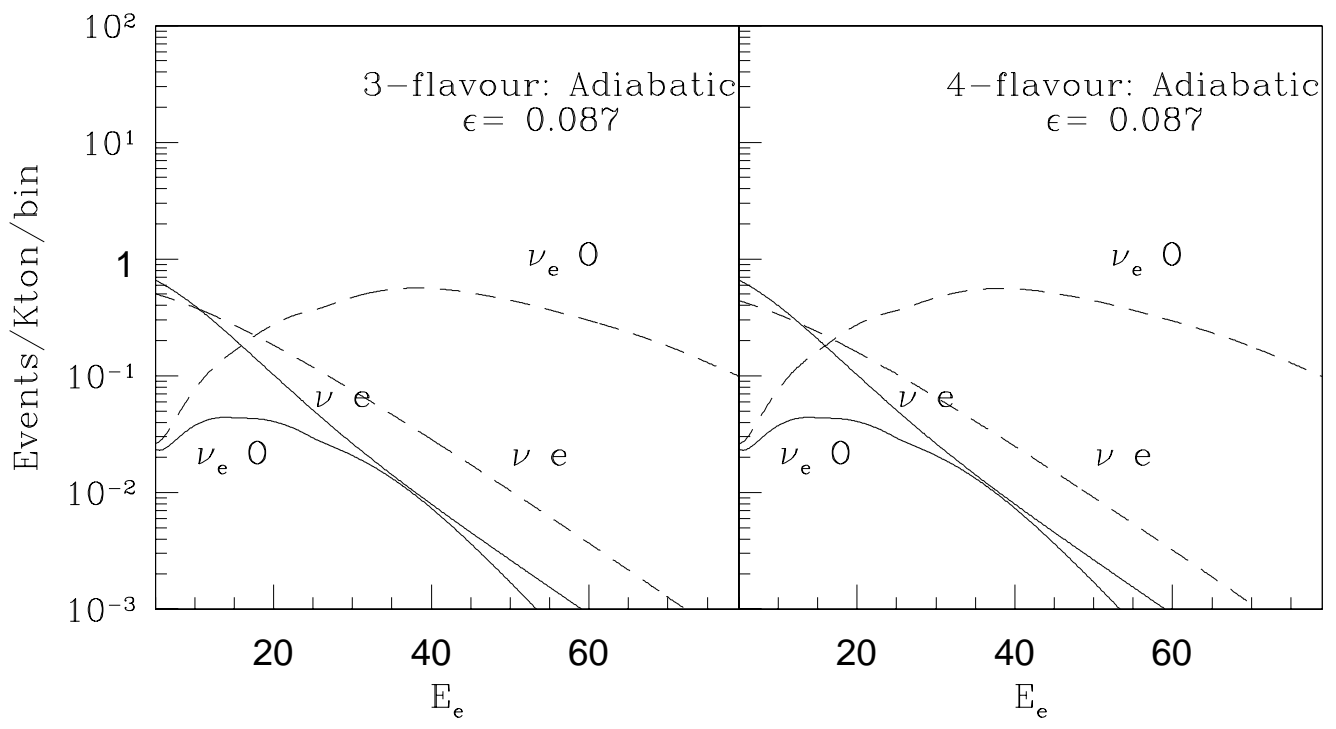

FIG. 3. $\nu_{e} O$ and $\nu e$ (for all flavours of $\nu$ ) event rates when the upper resonance is completely adiabatic. The solid lines represent the no mixing case and is plotted in all the graphs for comparison. The dashed lines are due to the effects of mixing. The oxygen events show dramatic increase due to mixing. Note that 3 and 4 flavour cases cannot be distinguished. 


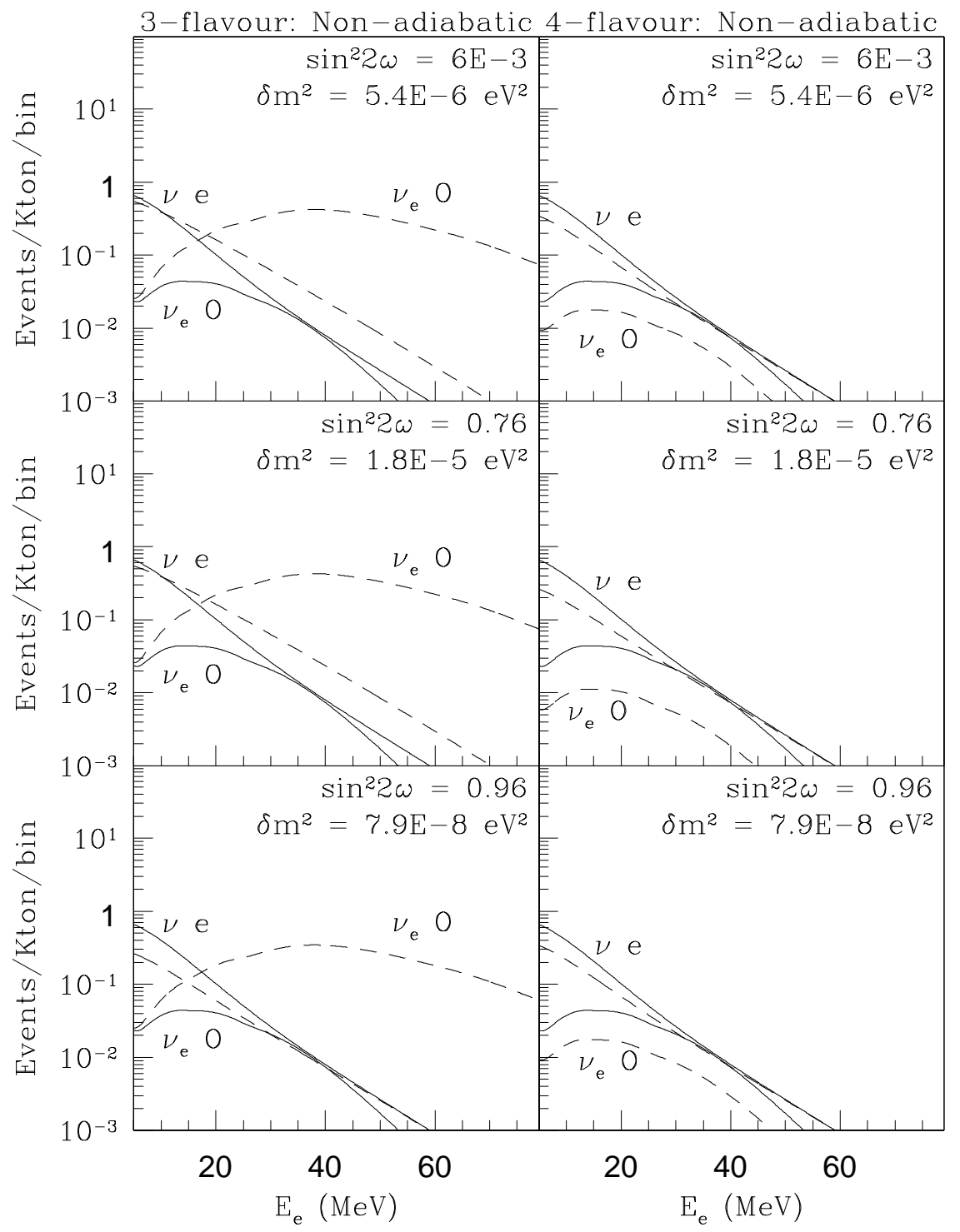

FIG. 4. $\nu_{e} O$ and $\nu e$ (for all flavours of $\nu$ ) event rates when the upper resonance is completely non-adiabatic. The results depend upon the three possible solutions to the solar neutrino puzzle and are shown in the three panels, top, middle and bottom. The three flavour results are similar to the adiabatic case shown in Fig. 3 but the 4 flavour case shows suppression of the event rates in all cases. Here the different cases are distinguished by the extent of suppression. 


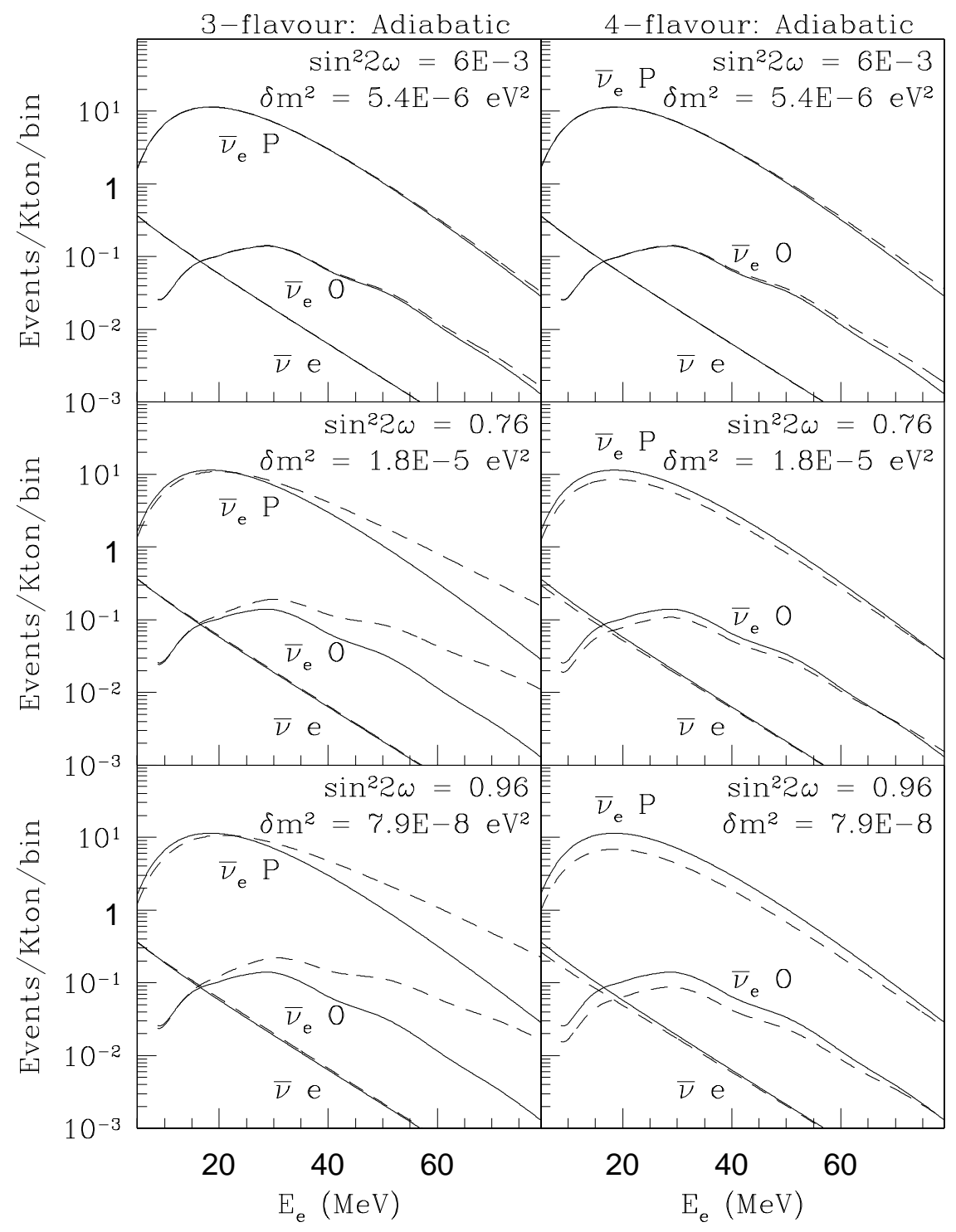

FIG. 5. $\overline{\nu_{e}}$ event rates for $\epsilon=0.087$. The solid (dashed) lines are due to (no) mixing. While the 3 flavour scheme shows enhancement of event rates at high energies due to mixing, the 4 flavour scheme shows suppression at lower energies. 